\title{
Uma análise de conteúdo sobre a percepção da qualidade de vida entre idosos residentes em um município sul baiano: Estudo Qualitativo
}

\author{
A content analysis on the perception of quality of life \\ among older adults resident in a southern municipality \\ of Bahia: Qualitative Study
}

\author{
Silas dos Santos Marques' ${ }^{1}$ (i) \\ Lina Faria ${ }^{2}$ (1) \\ Cristiano da Silveira Longo 3 (1)
}

\author{
${ }^{1}$ Autor para correspondência. Universidade Federal do Sul da Bahia (Itabuna). Bahia, Brasil. marx.fisio@gmail.com \\ ${ }^{2-3}$ Universidade Federal do Sul da Bahia (Porto Seguro). Bahia, Brasil. linafaria1964@gmail.com, cristianodasilveiralongo@gmail.com
}

RESUMO | INTRODUÇÃO: A mudança do perfil demográfico mundial proporcionado pelo aumento da expectativa de vida é um tema que vem suscitando discussões e investigações especialmente no que diz respeito à qualidade de vida (QV) na velhice. OBJETIVO: Avaliar a percepção de indivíduos idosos sobre o significado do envelhecimento em um município sul baiano e verificar associação entre as variáveis sociodemográficas e de saúde e a percepção da qualidade de vida. METODOLOGIA: Estudo transversal, descritivo, com abordagem qualitativa, realizado com 25 idosos no período entre março e maio de 2019, com base em dados coletados por questionário sociodemográfico e entrevista semiestruturada - gravada, transcrita e analisada mediante a Análise de Conteúdo. A amostra não probabilística contou com idosos com idade mínima de 60 anos que foram abordados nas salas de espera das Unidades Básicas de Saúde (UBS) e nos Grupos de Qualidade de Vida, atendidos pela equipe do Núcleo de Apoio à Saúde da Família (NASF) do Município. RESULTADOS: Da análise emergiram três categorias centrais: "Percepção sobre o processo de Envelhecimento"; Percepção sobre Qualidade de Vida" e "Motivos para satisfação com a Qualidade de Vida". Observou-se associação entre saúde, qualidade de vida, autonomia e boas relações familiares e sociais. Evidenciou-se que os idosos percebem e vivem de maneira ativa e entendem a autonomia como um desafio a ser enfrentado. CONCLUSÃO: Compreender como os idosos percebem o processo de envelhecimento, suas questões de saúde, relações sociais e convívio familiar, atrelados à noção de qualidade de vida, permite ao profissional de saúde pensar estratégias de cuidado para além do enfoque na doença, que incentivem ações específicas à esta população.

PALAVRAS-CHAVE: Envelhecimento. Qualidade de Vida. Autonomia.
ABSTRACT | INTRODUCTION: The change in the global demographic profile provided by the increase in life expectancy is a theme that has been causing discussions and investigations, especially with regard to the quality of life in old age. OBJECTIVE: Evaluate the perception of elderly individuals about the meaning of aging in a southern municipality of Bahia and verify the association between sociodemographic and health variables and the perception of quality of life. METHODOLOGY: Descriptive study with a qualitative approach, conducted with 25 elderly people between March and May 2019, based on data collected by a sociodemographic questionnaire and semi-structured interviews - recorded, transcribed, and analyzed through Content Analysis. The non-probabilistic sample was obtained, with elderly aged over 60 years who were approached in the waiting rooms of the Basic Health Units (UBS) and the Quality-of-life Groups, assisted by the family health support center team (NASF) of the municipality. RESULTS: Three central categories emerged from the analysis: "Perception about the aging process," "Perception about Quality of Life," and "Reasons for satisfaction with Quality of Life." There was an association between health, quality of life, personal autonomy, and good family and social relationships. The results suggest that older people perceive active aging and autonomy as a challenge to be faced. CONCLUSION: Understand how the elderly perceive the aging process and its health issues, social relationships, family life, linked to the notion of quality of life, allows the health professional to think strategies of care actions beyond the focus on the disease that encourage specific actions to seek changes in care and assistance to this population.

KEYWORDS: Aging. Quality of Life. Personal Autonomy. 


\section{Introdução}

O envelhecimento demográfico proporcionado pelo aumento da expectativa de vida criou novas demandas e desafios para as sociedades, as famílias cuidadoras e os governos dos diversos países. Contudo, em países como Portugal, Espanha, Alemanha, Japão, França o processo de envelhecimento ocorre associado às melhorias nas condições de vida desta faixa etária. Em países como o Brasil, esse processo vem ocorrendo de maneira acelerada sem reorganização dos serviços de saúde e propostas de políticas públicas que atendam às novas demandas emergentes. ${ }^{1}$

Cabe destacar como demandas e desafios o quadro sanitário atual provocado pelo Sars-Cov-2 (Covid-19), a tentativa de proteger os idosos em todo o mundo e os sistemas de saúde sobrecarregados. Neste cenário, emerge a necessidade de políticas públicas que incentivem o Envelhecimento Ativo e Redes de Suporte Familiar e Social que denotem as perspectivas e percepções dos indivíduos idosos e de suas famílias na busca e manutenção da Qualidade de Vida (QV).² Neste sentido, importante enfatizar o papel do NASF e a atuação integrada das equipes multiprofissionais com as equipes de Saúde da Família (ESF), no cuidado compartilhado e na construção conjunta de projetos terapêuticos de forma a ampliar o atendimento e o cuidado às populações idosas.

As dimensões autonomia e independência são fundamentais para a qualidade de vida e saúde..$^{3} \mathrm{~A}$ velhice põe em foco não somente questões relacionadas às qualidade de vida e à saúde, mas também as dimensões como autonomia e independência. Com o avançar da idade, há a prevalência de doenças crônicas e incapacitantes, as quais acarretam perda de autonomia, independência e dificuldades de agir por conta própria. 4,5

De acordo com a Organização Mundial da Saúde $(\mathrm{OMS})^{6}$, o conceito de Qualidade de Vida deve ser entendido por meio da percepção do indivíduo sobre sua posição nos sistemas de valores nos quais vive e em relação aos seus objetivos, expectativas, subjetividades e preocupações. É um conceito necessário na prática dos cuidados e nas pesquisas em saúde e vem sendo utilizado por clínicos, pesquisadores, economistas, administradores e políticos para entender as dimensões como autonomia e independência. $\underline{7}$

No Brasil, as regiões Sudeste e Nordeste apresentam os maiores percentuais de população idosa do território nacional, com 42,17\% e 27,17\%, respectivamente, segundo dados da Pesquisa Nacional por Amostra de Domiciliados Contínua (PNADC) de 2019. No Nordeste, esse percentual explica-se por motivos relacionados à queda acelerada da fecundidade e da mortalidade.. A Pesquisa Nacional de Amostra por Domicílio realizada em 2015, apresentou um aumento $3,4 \%$ em cinco anos, totalizando um percentual de $13,4 \%$ de idosos na população baiana. ${ }^{9}$ No município baiano de Eunápolis, foco do presente estudo, o relatório do E-SUS de 2018 indica 88.996 idosos cadastrados na atenção básica, dentre os quais 11.202 possuem idades iguais ou superiores há 60 anos. Deste total, 2.036 são considerados idosos longevos e 9.166 idosos jovens.

Neste sentido, com base nos achados sobre o cenário do processo de envelhecimento no Brasil e no mundo, e também sobre a realidade do estado da Bahia, com aumento significativo da população idosa nas últimas décadas, o presente estudo busca investigar a percepção sobre a velhice, relacionando-a à situação de saúde e à qualidade de vida de indivíduos idosos participantes dos "Grupos de Qualidade de Vida" de uma equipe do NASF do Município de Eunápolis-Ba.

\section{Métodos}

Trata-se de um estudo transversal, qualitativo, desenvolvido com base na análise de conteúdo, realizado em um município sul baiano, Eunápolis, no período entre março e maio de 2019, em dados coletados por questionário sociodemográfico e entrevista semiestruturada sobre os temas estudados nesta pesquisa. O presente estudo foi aprovado pelo Comitê de Ética em Pesquisa da Universidade Federal do Sul da Bahia (UFSB) com número de registro: 03357318.4.0000.8467.

Importante destacar a pesquisa do Instituto Brasileiro de Geografia e Estatística (IBGE), publicada em agosto de 2019, que aponta o aumento da população idosa e a queda no número de nascimentos no estado da Bahia. Ainda segundo a pesquisa, em 2013, a população que tinha até 13 anos era de $22,6 \%$. Esse número caiu para 19,2\% em 2019. Em contrapartida, o índice de baianos com mais de 60 anos foi de $12 \%$ para $15,2 \%$. No caso específico do município estudado, também se observou o aumento do número de idosos de acordo com os dados do E-SUS 2018. 
Este estudo contou com uma casuística composta por 25 idosos que, inicialmente, foram selecionados em oito grupos de convivência, denominados "Grupos de Qualidade de Vida", assistidos pela Equipe do Núcleo de Apoio à Saúde da Família (NASF) do município de Eunápolis. Os Grupos de Qualidade de Vida acontecem em duas Unidades Básicas de Saúde (UBS) do município -- Urbis e Rosa Neto. Contudo, em função da baixa participação de idosos nos grupos de convivência do NASF, houve necessidade de incluir outros participantes para a coleta de dados. Neste sentido, foram aplicados também questionários para idosos presentes nas salas de espera das duas UBS, que manifestaram interesse em participar da pesquisa. Dos 25 idosos que fizeram parte deste estudo, 7 participam dos Grupos de Qualidade de Vida - 2 idosos da UBS Urbis e 6 idosos da UBS Rosa Neto -, e 18 foram abordados nas salas de espera para a aplicação dos questionários - 6 idosos da UBS Urbis e 12 idosos da UBS Rosa Neto.

A amostra foi obtida de forma não probabilítica, contando com idosos com idade mínima de 60 anos, de ambos os gêneros e cognitivamente capazes para responder às questões, que foram abordados nos Grupos de Qualidade de Vida e nas salas de espera das duas UBS.

Dentre os idosos com idades inferiores a 80 anos, no município de Eunápolis, 4.884 são do sexo feminino e 4.282 do sexo masculino. Para auxiliar no serviço de assistência à saúde, o município dispõe de 30 equipes da ESF distribuídas em 25 Unidades Básica de Saúde (UBS) e 3 equipes de PACS (Programa de Agentes Comunitários de Saúde), com uma equipe itinerante, que atua principalmente na zona rural.

A rede de saúde do município estudado incentiva Programas de melhoria da qualidade de vida para a sociedade de modo geral, com ações direcionadas para os diversos grupos. Com essa finalidade, os Grupos de Qualidade de Vida reúnem-se nas duas unidades cobertas pelas equipes do NASF, que oferecem programações para o autocuidado, além de orientações sobre hábitos saudáveis, envelhecimento ativo e redução dos impactos da velhice.

O questionário sociodemográfico, para caracterização da amostra, foi composto por oito questões, que abordaram informações sobre: dados pessoais; grupo familiar; trabalho; renda e condições de moradia. Cabe destacar que o questionário foi adaptado dos questionários utilizados no Estudo Longitudinal da Saúde do Idoso (ELSI-Brasil), organizado pelo Ministério da Saúde em parceria com a Fundação Oswaldo Cruz (FIOCRUZ). ${ }^{10}$

O roteiro da entrevista (semiestruturada) foi composto por quatro questões abertas sobre a percepção dos idosos do processo de envelhecimento e sua qualidade de vida - o que significa envelhecer pra você? O que é qualidade de vida na terceira idade? Como você avalia sua qualidade de vida? Gostaria de acrescentar algo mais sobre o tema qualidade de vida e envelhecimento? As questões foram gravadas em um aparelho celular para armazenamento e posterior análise. Em seguida, as gravações foram transcritas na íntegra e analisadas, de acordo com a análise de conteúdo proposta por Bardin. ${ }^{11}$ Foram seguidas etapas descritas como pré-análise, descrição analítica e interpretação inferencial do conteúdo obtido. Na primeira etapa, foi realizada leitura exploratória por meio da relação das respostas com a temática sob a qual cada pergunta foi elaborada. Em seguida, na fase de descrição analítica, a organização e sistematização dos dados por categorias e subcategorias de análises, e por fim as unidades significantes a partir dos relatos categorizados de acordo com os significados identificados nas entrevistas. Para a discussão e apresentação dos resultados, as falas foram numeradas de 1 a 25 , e a letra "l" foi utilizada para representar "idoso".

\section{Resultados}

A tabela 1 apresenta o perfil sociodemográfico da amostra estudada. A amostra estudada, que apresentou idade média de 69 anos, sendo 4 do sexo masculino e 21 do sexo feminino. Deste total, 13 idosos declararam terem residido em áreas rurais, em algum momento de sua vida. A tabela 1 , mostra o percentual igual entre casados e viúvos. Em relação à cor, 12 participantes se autodeclararam pardos, e em relação ao número de filhos, a quantidade média foi de 3 filhos por indivíduo. Chama a atenção o fato de apenas 8 participantes terem ensino médio completo e um único caso ensino superior completo. Chama a atenção também o fato de o sexo feminino ser fator preditor de maior busca por assistência à saúde. 
Tabela 1. Perfil Sociodemográfico dos idosos de Eunápolis, BA. 2019

\begin{tabular}{|c|c|c|c|}
\hline \multicolumn{4}{|c|}{ Quantidade de Filhos } \\
\hline & Média & \multicolumn{2}{|l|}{ DP } \\
\hline & 3 & \multicolumn{2}{|l|}{2} \\
\hline \multicolumn{4}{|c|}{ Idade } \\
\hline & Média & \multicolumn{2}{|l|}{ DP } \\
\hline & 69 & \multicolumn{2}{|l|}{5,6} \\
\hline \multicolumn{4}{|c|}{ Sexo } \\
\hline & Frequência & \multicolumn{2}{|l|}{ Porcentual } \\
\hline Masculino & 4 & & 16,0 \\
\hline Feminino & 21 & & 84,0 \\
\hline \multicolumn{4}{|c|}{ Moradia } \\
\hline \multicolumn{2}{|c|}{ Frequência } & \multicolumn{2}{|l|}{ Porcentual } \\
\hline Morou em zona rural & 13 & & 52,0 \\
\hline Não morou em zona rural & 12 & & 48,0 \\
\hline \multicolumn{4}{|c|}{ Estado Civil } \\
\hline \multicolumn{2}{|c|}{ Frequência } & \multicolumn{2}{|l|}{ Porcentual } \\
\hline Casado(a)/Amasiado(a)/União estável & 11 & & 44,0 \\
\hline Divorciado(a)/Separado(a) & 3 & & 12,0 \\
\hline Viúvo(a) & 11 & & 44,0 \\
\hline \multicolumn{4}{|c|}{ Cor } \\
\hline \multicolumn{2}{|r|}{ Frequência } & \multicolumn{2}{|l|}{ Porcentual } \\
\hline Branca & 6 & & 24,0 \\
\hline Preta & 6 & & 24,0 \\
\hline Parda & 12 & & 48,0 \\
\hline Amarela & 1 & & 4,0 \\
\hline \multicolumn{4}{|c|}{ Escolaridade } \\
\hline \multicolumn{2}{|r|}{ Frequência } & \multicolumn{2}{|l|}{ Porcentual } \\
\hline Nunca estudou & 3 & & 12,0 \\
\hline Primário incompleto & 5 & & 20,0 \\
\hline Primário completo & 4 & & 16,0 \\
\hline Ginásio completo & 4 & & 16,0 \\
\hline Ensino médio completo & 8 & & 32,0 \\
\hline Curso superior & 1 & & 4,0 \\
\hline
\end{tabular}

*DP - Desvio Padrão

Fonte: Dados dos próprios autores 
No Quadro 1 estão elencadas as unidades significantes e a categorização realizada a partir dos relatos dos participantes, após a gravação, transcrição e análise dos conteúdos. A partir de então, foram apresentadas de acordo com o tema abordado em cada etapa que compõe o questionário semiestruturado utilizado para a obtenção dos referidos dados.

Quadro 1. Unidade Significante, Categorias e Subcategorias da percepção sobre envelhecimento e Qualidade de vida dos idosos de Eunápolis, BA. 2019

\begin{tabular}{|c|c|c|c|}
\hline UNIDADE SIGNIFICANTE & CATEGORIA & SUBCATEGORIA & TEMA \\
\hline $\begin{array}{l}\text { Envelhecer como privilégio; } \\
\text { Envelhecimento como } \\
\text { processo cronológico e natural; } \\
\text { Envelhecimento e } \\
\text { aprendizagem; }\end{array}$ & $\begin{array}{l}\text { Percepção sobre o } \\
\text { Processo de } \\
\text { Envelhecimento }\end{array}$ & $\begin{array}{c}\text { Sentimento em relação ao } \\
\text { envelhecimento }\end{array}$ & \multirow{2}{*}{$\begin{array}{l}\text { Significado de } \\
\text { envelhecimento }\end{array}$} \\
\hline $\begin{array}{c}\text { Necessidade de Autocuidado; } \\
\text { medo de depender de outras } \\
\text { pessoas; insatisfação com a } \\
\text { velhice. }\end{array}$ & $\begin{array}{l}\text { Vulnerabilidades e } \\
\text { Fragilidades na velhice }\end{array}$ & $\begin{array}{l}\text { Surgimento de doenças com } \\
\text { perda de autonomia e da } \\
\text { funcionalidade. }\end{array}$ & \\
\hline $\begin{array}{c}\text { Perdas sociais, relações } \\
\text { familiares; continuidade do } \\
\text { cuidado. }\end{array}$ & $\begin{array}{l}\text { Percepção sobre } \\
\text { Qualidade de Vida }\end{array}$ & $\begin{array}{l}\text { Acesso aos serviços de saúde; } \\
\text { Participação Social; } \\
\text { Relação com os profissionais } \\
\text { de saúde }\end{array}$ & \multirow{2}{*}{$\begin{array}{c}\text { Conceitos de qualidade } \\
\text { de vida no } \\
\text { envelhecimento }\end{array}$} \\
\hline $\begin{array}{l}\text { Lazer; Condição financeira para } \\
\text { se cuidar. }\end{array}$ & $\begin{array}{l}\text { Percepção sobre o Bem- } \\
\text { estar Psicossocial; }\end{array}$ & $\begin{array}{l}\text { Manter uma vida tranquila } \\
\text { para viver melhor. }\end{array}$ & \\
\hline $\begin{array}{c}\text { Manutenção das s relações } \\
\text { interpessoais e de hábitos } \\
\text { saudáveis. }\end{array}$ & $\begin{array}{c}\text { Motivos para Satisfação } \\
\text { com a Qualidade de } \\
\text { Vida. }\end{array}$ & $\begin{array}{c}\text { Qualidade de vida e } \\
\text { condições econômicas e } \\
\text { sociais }\end{array}$ & \multirow{2}{*}{$\begin{array}{l}\text { Auto avaliação da } \\
\text { Qualidade de Vida. }\end{array}$} \\
\hline $\begin{array}{c}\text { Distância da família; Solidão; } \\
\text { Redução da capacidade para o } \\
\text { trabalho; }\end{array}$ & $\begin{array}{l}\text { Motivos para } \\
\text { insatisfação com a } \\
\text { Qualidade de Vida. }\end{array}$ & $\begin{array}{c}\text { A família como determinante } \\
\text { na Qualidade de Vida }\end{array}$ & \\
\hline
\end{tabular}

\section{Significado de Envelhecimento}

Sobre a percepção dos idosos do processo de envelhecimento, verificou-se a categoria "Percepção sobre o processo de Envelhecimento" e a subcategoria "Sentimento em relação ao envelhecimento". A partir de então, foram observadas as unidades significantes presentes nos relatos. No relato de I 10: "Pra mim? Foi a melhor coisa do mundo, eu que vivi mais velha do que eu vivi nova, só a experiência que a gente pode passar, pra mim já valeu. Eu tenho 78 anos, então esse aprendizado vai me ajudar muito para o resto da vida". Por meio desta fala, assim como em outras falas relacionadas a este primeiro tema, é possível extrair significados do envelhecimento como um privilégio e oportunidade de aprendizado.

O relato de I 19 demonstra outra perspectiva sobre o significado do envelhecimento, como um evento cronológico e natural, que pode ser observado quando diz: "... é a pessoa que viveu que já viveu bastante e se tornou uma pessoa idosa".

Em contrapartida, ainda existem outros aspectos considerados por muitos idosos investigados relacionados às fragilidades com a idade avançada - Categoria de "Vulnerabilidade e Fragilidade na Velhice", com a subcategoria - "surgimento de doenças com perda de autonomia e da funcionalidade". Essas percepções aparecem nas falas de I 16: "... a velhice não é muito bom pra mim não". Denota um significado de insatisfação com essa nova fase; 
bem como na fala de 113: "Envelhecer, é essas doenças todas que eu tenho tido"; e na fala de I 22: "Pra mim é quando a pessoa não pode mais andar", que exprime a ideia de um envelhecimento intimamente ligado ao adoecimento e, consequentemente, à dependência e perda de funções essenciais para autonomia.

\section{Conceitos de Qualidade de Vida no envelhecimento}

Na categoria estabelecida como "Percepção sobre qualidade de vida", as subcategorias "acesso aos serviços de saúde", "participação social" e "relação com os profissionais de saúde" fazem emergir de suas falas significados que denotam perdas sociais, como no relato de I 9: "Qualidade de vida é a pessoa ser simples, viver independente, ter condição, e aí se ele tem condição ele tem qualidade de vida". Quando I 22 diz: "...é você estar bem, estar bem com você mesmo, estar bem com aquele que convive ao seu lado. É isso aí." Enfatiza o significado de que qualidade de vida está ligada a viver bem com a família. Mas a preocupação com a saúde expressa a preocupação com o significado de continuidade do cuidado, como, por exemplo, quando I 15 relata: "...É ter condições assim, pra se cuidar né?", e ainda 116: "Pra mim não é muito boa não. Eu sinto muitas dores. Eu sinto muitas dores depois de velha".

Na categoria "Bem-estar psicossocial" e na subcategoria "Manter uma vida tranquila para viver melhor", o relato de I 13, enfatiza a importância do lazer para manutenção da qualidade de vida: "Qualidade de Vida é curtir, passear, andar, viajar e tudo. Tenho dinheiro, mas não tenho a saúde...". Em contrapartida, 115 ao relatar: "É ter condições assim, pra se cuidar né? Eu acho que essa é a melhor qualidade que tem. Uma boa alimentação, ter condições pra ir vivendo", traz consigo a ideia de que não dá para ter qualidade de vida se as condições de cuidado são precárias.

\section{Auto avaliação da Qualidade de Vida}

Quando convidados a expressar a autoavaliação da qualidade de vida, os idosos apresentaram respostas antagônicas; alguns se consideravam satisfeitos e outros não. Emergiu dessas impressões a Categoria "Motivos para satisfação com a Qualidade de Vida", que, por sua vez, auxilia na observação da subcategoria "Qualidade de vida atrelada às condições econômicas e sociais". Desta categoria derivouse os significados na melhora progressiva que já ocorreu ou que deve ocorrer, como no relato de 15:
"Por tudo que eu já passei na vida tá boa agora", e de I 3: "Bem, na idade que estou, eu acho que está ótima...". Expressa ainda a necessidade de estar bem com as pessoas à sua volta, valorizando o significado de boas relações sociais, como relatado por I 8: "Normal, assim né, porque amo meus filhos de coração, meus netos, é boa demais essa minha vida. Amo todo, mundo nunca briguei com ninguém, nunca discuti com ninguém, as pessoas dizem as coisas assim, eu tenho é que rezar por elas, porque o encardido é feio". E, partindo dessa categoria, tem-se ainda o significado dos bons hábitos como um fator influenciador da qualidade de vida, expresso por I 18: "...Mas de um ano e meio, eu estou bem com minha alimentação, dormindo mais cedo, então pra mim, de um ano e meio pra cá, teve diferença".

A auto avaliação da Qualidade de Vida fez surgir também em meio aos relatos dos entrevistados a Categoria "Motivos para a insatisfação com a qualidade de vida" e a subcategoria "A família como determinante na Qualidade de Vida". A tristeza com a distância da família fica explícita em alguns relatos como de I 2: "A minha é boa, mas poderia ser melhor, se eu estivesse perto dos meus filhos, por que eles moram meio longe, os meus netos. Então, já fica meio triste", e de 1 7: "Eu acho boa, assim, em uns pontos né. Bom em uns pontos e em outro ruim. Porque assim, eu moro sozinha...". A capacidade para o trabalho também surge como importante significado para a qualidade de vida, I 9: "...Já foi boa, hoje é regular, porque hoje eu não posso trabalhar, não tenho recurso, mas tenho aquilo que posso".

\section{Discussão}

Alguns estudos têm indicado o impacto da incapacidade funcional na vida diária de idosos ${ }^{12}$ ao observarem que o perfil sociodemográfico desta faixa etária se assemelha à média nacional, com a presença majoritária do sexo feminino, idade média de 71 anos, casados e com escolaridade entre ensino fundamental (completo ou não) e médio. Estes resultados se assemelham com os encontrados na presente pesquisa: observou-se idade média de 69 anos, com maioria do sexo feminino, com números iguais entre casados(a) e viúvos(a) e escolaridade mais frequente de ensino fundamental I e II (completo ou não).

O presente estudo possibilitou observar que a experiência do envelhecimento é bem particular, e que 
para cada indivíduo a visão é construída de acordo com suas vivências e percepções sobre saúde e doença, uma vez que, os idosos ao serem indagados sobre o significado de envelhecer, apresentaram opiniões diferentes: alguns idosos demonstraram que se sentem privilegiados por terem envelhecido, e que com o passar dos anos adquiriram experiência, e aprenderam a viver. Meneses et al. $\frac{13}{3}$ corroboram esses achados, pois, ao entrevistarem 12 idosos, encontraram relatos que exprimem a ideia de que para esses idosos a velhice pode ser traduzida em ganhos e oportunidade, representando um momento de compartilhamento das experiências de vida e de conhecimentos acumulados ao longo dos anos.

O significado de envelhecimento para os idosos estudados apresenta-se associado às perdas das capacidades físicas, demonstrado por meio de relatos como o de I 22: "Velhice pra mim é quando a pessoas não pode mais andar". O que é semelhante ao encontrado no trabalho de Brunnet et al. ${ }^{14}$, que observaram que para muitos idosos "Envelhecer" estava associado à impossibilitadas de exercer alguma atividade mediante o envelhecimento do corpo e/ou da mente. Teixeira e Neri ${ }^{15}$ enfatizam que a capacidade funcional, satisfação com a vida, longevidade, ausência de incapacidade, domínio/crescimento, participação social ativa, alta capacidade funcional/independência e adaptação positiva, devem ser consideradas no processo de envelhecimento.

Quanto à percepção da qualidade de vida no enveIhecimento, Cartaxo et al. ${ }^{16}$ afirmam que, a partir de resultados de pesquisa, conseguiram observar que $o$ domínio do meio ambiente foi referido, com maior frequência, como influenciador da qualidade de vida. Contudo, a saúde e a independência financeira apresentam-se como fatores importantes para a Qualidade de vida, bem como as atividades de Lazer. No presente estudo, foi muito recorrente a referência às condições financeiras, sempre associadas à saúde, representando uma ideia de interdependência da saúde e a situação financeira, que por vezes apresentou-se como desfavorável durante as falas dos idosos entrevistados. Demonstram ainda preocupação com as morbidades já instaladas e as possibilidades de lazer durante esta fase da vida.

Alves et al. ${ }^{17}$ captaram, a partir da entrevista de 10 idosos, que a qualidade de vida além de estar relacionada ao estado de saúde, assim como no presente estudo, relaciona-se também a um "bom salário", para gerar melhor acesso aos serviços de saúde, prática de atividade física e manutenção da alimentação saudável. Essa preocupação, tão recorrente, pode ser melhor compreendida em estudos ${ }^{18}$ que reiteram que o envelhecimento, naturalmente, conduz os indivíduos à uma certa fragilidade que lhes colocam susceptíveis a desequilíbrios no processo saúde-doença, gerando a necessidade de cuidados especiais. Nesta realidade, por vezes, acabam por vivenciarem situação de instabilidade financeira, na medida em que sua renda se torna insuficiente para lhes possibilitar os cuidados, muitas vezes especiais, que necessitam.

O conceito de "autoeficácia" $\underline{19}$, ou seja, a capacidade do indivíduo lidar com as exigências externas da vida diária, tem como componente principal as experiências bem-sucedidas, e sua importância resulta do fato de ter como base experiências reais vivenciadas pelos próprios indivíduos. Correlacionando com este conceito, os idosos demonstram ter sentimentos negativos relacionados à autoeficácia, traduzindose pela importância dada à situação financeira aliada aos problemas inerentes à velhice. Esta situação pode ser explicada quando Martins e Borges ${ }^{20}$ tratam da aposentadoria e afirmam que a aposentadoria se constitui um marco de transição de uma vida com rotina atrelada ao trabalho, para uma nova etapa marcada por perdas, o que pode desencadear crises.

Na população idosa, a qualidade de vida abrange vários aspectos - nível socioeconômico, interação social, atividade intelectual, autocuidado, estado de saúde, valores culturais, éticos, religiosidade, capacidade funcional e suporte familiar ${ }^{21}$. Neste sentido, Oliveira et al.22, em um estudo sobre o impacto do treinamento funcional coletivo na qualidade de vida de idosos, indicaram a percepção da qualidade de vida atrelada ao convívio social. Neste sentido, fundamental o incentivo à políticas públicas direcionadas para o fortalecimento de redes formais e informais de apoio à esta faixa etária. $\underline{11}$

O apoio familiar e a espiritualidade foram observados como fatores fundamentais para a maioria dos idosos na manutenção do equilíbrio emocional e como forma de enfrentar a dependência física e econômica. $\underline{23}$ Neste sentido, quando indagados sobre a autoavaliação da qualidade de vida, os idosos apresentaram visões heterogêneas. Importante destacar que a motivação para uma avaliação positiva ou negativa é multifatorial, podendo ser influenciada por fatores intrínsecos e extrínsecos 
relacionados ao estado de saúde, ao autocuidado, às dificuldades superadas e às relações familiares. Corroborando com este estudo, Pereira et al. ${ }^{24}$ demonstraram que os idosos, de modo geral, se auto avaliaram como satisfeitos com as condições de vida a insatisfação tem relação com os problemas de saúde.

Conforme os resultados apresentados, qualidade de vida é, principalmente, viver bem, com autonomia e saúde, conviver com a família e amigos, poder realizar atividades de lazer e ter recursos para manter suas necessidades. Para os idosos, QV constitui-se num construto multidimensional, e que as percepções têm significados específicos para essa faixa etária. Contudo, o trabalho apresenta algumas limitações, como o tamanho da amostra, não sendo possível verificar significância em algumas análises.

\section{Conclusão}

O envelhecimento é um fenômeno mundial experimentado, sentido e percebido de forma particular por cada indivíduo, de acordo com sua vivência durante os ciclos de vida. A partir desses resultados, pode-se concluir que a QV dos idosos nas duas unidades básicas de saúde Urbis e Rosa Neto nos Grupos de Qualidade de Vida, assistidos pela Equipe do NASF do município de Eunápolis apresentou, em geral, resultados positivos. A autoavaliação da QV com base em dados coletados por questionário sociodemográfico e entrevista semiestruturada evidencia que os idosos participantes deste estudo estavam satisfeitos com sua QV. Os domínios de relações sociais com familiares e amigos e da autonomia se destacaram dos outros domínios.

A autonomia é uma questão importante na atenção e no cuidado ao idoso pelas equipes do NASF e da ESF, na medida que é central para o envelhecimento saudável e a manutenção da QV em geral, uma vez que está relacionada às condições cognitivas na velhice.

Estudos sobre percepção do idoso sobre QV devem ser incentivados, assim como amostragem probabilística e coortes observacionais, de forma que essa percepção possa ser explorada. Sendo assim, sugerese intervenções que possibilitem impactos positivos, uma vez que o conceito ampliado de saúde sugere a qualidade de vida como elemento fundamental no processo saúde-doença-cuidado.

\section{Contribuições dos autores}

Marques SS participou da construção do projeto, coleta e análise de dados e na construção do texto final. Faria L orientadora do trabalho, apoiou no planejamento do estudo, orientou a coleta e interpretação de dados, bem como apoiou na redação e revisão final do artigo. Longo CS participou da redação e revisão do artigo cientifico.

\section{Conflitos de interesses}

Nenhum conflito financeiro, legal ou político envolvendo terceiros (governo, empresas e fundações privadas, etc.) foi declarado para nenhum aspecto do trabalho submetido (incluindo, mas não se limitando a subvenções e financiamentos, participação em conselho consultivo, desenho de estudo, preparação de manuscrito, análise estatística, etc.).

\section{Referências}

1. Faria LR, Santos LAC. Sense of Coherence: O sentido de coerência nos caminhos do envelhecimento. In: Faria LR, Calábria LK, Alves WA, organizadores. Envelhecimento: Um olhar interdisciplinar. São Paulo: Hitec; 2016. p. 19-42.

\begin{abstract}
2. Matos AMDA, Perufo KF. "O mais importante é ter saúde": Representações sociais sobre o envelhecimento positivo. In: Faria LR, Calábria LK, Alves WA. Envelhecimento: Um olhar interdisciplinar. São Paulo: Hitec; 2016. p. 43-60.
\end{abstract}

3. Martins CRM, Camargo BV, Biasus F. Representações sociais do idoso e da velhice de diferentes faixas etárias. Univ Psychol [Internet]. 2009;8(3):831-47. Disponível em: http://www.redalyc. org/articulo.oa?id $=64712155020$

4. Azevedo ALS, Silva RA, Tomasi E, Quevedo LA. Doenças crônicas e qualidade de vida na atenção primária à saúde. Cad. saúde pública. 2013;29(9):1774-82. https://doi.org/10.1590/0102$\underline{311 \times 00134812}$

5. Faria LR. Atenção preventiva e educativa em saúde do idoso: o saber e fazer compartilhados [Internet]. Juiz de Fora: Revista A3; 2015. Disponível em: http://www.ufjf.br/ revistaa3/2015/10/05/1017/

6. Organização Mundial da Saúde. Manual da Classificação Estatística Internacional de Doenças, Lesões e Causas de Morte [Internet]. São Paulo: OMS; 1948. Disponível em: https://apps. who.int/iris/bitstream/handle/10665/70943/ICD_10_1980_v1_pt_1. pdf? sequence $=4 \&$ isAllowed $=y$ 
7. Fleck MPA. A avaliação da qualidade de vida: guia para profissionais da saúde. Porto Alegre: Artmed; 2008.

8. Instituto Brasileiro de Geografia e Estatística. Pesquisa Nacional por Amostra de Domicílios Contínua -PNADC [Internet]. 2020 [acesso em 2021 Mai 10]. Disponível em: https://sidra.ibge.gov.br/ tabela/6407\#resultado

9. Instituto Brasileiro de Geografia e Estatística. Pesquisa nacional por amostra de domicílio: síntese de indicadores 2015 [Internet]. Rio de Janeiro: IBGE; 2016. Disponível em: https://biblioteca.ibge. gov.br/visualizacao/livros/liv98887.pdf

10. Lima-Costa MF. Envelhecimento e saúde coletiva: Estudo Longitudinal da Saúde dos Idosos Brasileiros (ELSI-Brasil). Rev Saude pública. 2018;52(Suppl 2):2s. https://doi.org/10.11606/ S1518-8787.201805200supl2ap

11. Longo CS, Narita S. "Um Corpus que Fala”: Apontamentos para uma Revisão Técnica da Análise de Conteúdo. Web-Revista Sociodialeto [Internet]. 2014;4(12):42-60. Disponível em: https:// ptdocz.com/doc/570785/um-corpus-que-fala---web-revistasociodialeto

12. Santos LAC, Faria LR, Patino RA. O envelhecer e a morte: leituras contemporâneas de psicologia social. Rev. bras. Est. Pop. 2018;35(2):e0040. http://dx.doi.org/10.20947/s0102-3098a0040

13. Meneses DLP, Silva Júnior FJG, Melo HSF, Silva JC, Luz VLES, Figueiredo MLF. A dupla face da velhice: o olhar de idosos sobre o processo de envelhecimento. Enferm foco. 2013;4(1):15-8. https:// doi.org/10.21675/2357-707X.2013.v4.n1.495

14. Teixeira INDO, Neri AL. Envelhecimento bem-sucedido: uma meta no curso da vida. Psicol. USP. 2008;19(1):81-94. http://dx.doi. org/10.1590/S0103-65642008000100010

15. Brunnet AE, Andrades B, Souza CS, Weber JLA, Martinato L, Loreto $\mathrm{T}$, et al. Práticas sociais e significados do envelhecimento para mulheres idosas. Pensando fam [Internet]. 2013;17(1):99109. Disponível em: http://pepsic.bvsalud.org/scielo. php? script=sci_arttext\&pid=S1679-494X2013000100010\&lng=pt\& $\underline{\mathrm{nrm}=\mathrm{iso}}$

16. Cartaxo HGO, Silva EAPC, Santos ARM, Siqueira PGBS, Pazzola CM, Freitas CMSM. Percepção de idosas sobre o envelhecimento com qualidade de vida: subsídio para intervenções públicas. Rev Rene [Internet]. 2012;13(1):158-68. Disponível em: http://www. periodicos.ufc.br/rene/article/view/3787/2992
17. Alves ERP, Dias MD, Costa AM, Silva ARS, Silva MM, Seabra RV. Qualidade de Vida: Percepção de Idosos de uma Unidade de Saúde da Família. Rev Enferm UFSM [Internet]. 2012;2(3):487-95. Disponível em: https://periodicos.ufsm.br/index.php/reufsm/ article/view/5240/pdf

18. Lopes MJ, Araújo JL, Nascimento EGC. O envelhecimento e a qualidade de vida: a influência das experiências individuais. Revista Kairós Gerontologia [Internet]. 2016;19(2):181-99. Disponível em: https://revistas.pucsp.br/index.php/kairos/article/ view/32155/22221

19. Cruz C, Navarro-Pardo E, Pocinho R, Anjos VN, Jacob L. A auto-eficácia na adaptação aos desafios do envelhecimento. Rev. Lusofona de Educ. [Internet]. 2017;38:181-94. Disponível em: http://revistas.ulusofona.pt/index.php/rleducacao/article/ view/6268

20. Martins LF, Borges ES. Educação para aposentadoria: avaliação dos impactos de um programa para melhorar qualidade de vida pós-trabalho. Interações. 2017;18(3):55-68. https://doi. org/10.20435/inter.v18i3.1496

21. Faria L, Alves WA, Amaral S. Sentimento mútuo: as relações de cuidar e ser cuidado no domicílio. In: Faria LR, Calábria LK, Alves WA, organizadores. Envelhecimento: Um olhar interdisciplinar. São Paulo: Hitec; 2016

22. Oliveira DV, Silva MR, Freire GLM, Batista RPR, Nascimento Junior JRA. Condições de saúde e qualidade de vida de idosos praticantes de treinamento funcional. R Bras Qual Vida [Internet]. 2019;11(4):e10359. Disponível em: https://periodicos.utfpr.edu.br/ rbqv/article/view/10359/pdf

23. Tavares KO, Scalco JC, Vieira L, Silva JR, Bastos CCCB. Envelhecer, adoecer e tornar-se dependente: a visão do idoso. Revista Kairós Gerontologia [Internet]. 2013;15(3):10518. Disponível em: https://revistas.pucsp.br/kairos/article/ download/8979/10186

24. Pereira KG, Silva PLN, Oliveira MKS, Gamba MA, Alves ECS, Martins AG. Autoavaliação da saúde por idosos atendidos em um centro ambulatorial de referência. J Manag Prim Health Care. 2018;9:e5. https://doi.org/10.14295/jmphc.v9i0.434 